\title{
Índice de Salud Prostática (phi)
}

\section{Prostate Health Index (phi)}

Ana Isabel Toro-Montoya1 (iD, Jennifer C. Vizcaíno-Carruyo² (iD,

Natalia M. Guevara-Arismendy ${ }^{3}$ iD, Germán Campuzano-Zuluaga ${ }^{4}$ iD

\section{Utilidad clínica de la prueba}

En Colombia, el cáncer de próstata es una de las principales causas de mortalidad por cáncer, el cual se ha triplicado durante los últimos años, con una incidencia anual de 14.460 casos y una mortalidad de 3.846 durante el 2020 [1].

El antígeno específico de próstata (PSA, del inglés, Prostate Specific Antigen) es una glicoproteína producida por la próstata, y es el marcador tumoral de mayor uso. Sin embargo, su baja especificidad para diferenciar entre cáncer de próstata y otras alteraciones no malignas, como la hipertrofia benigna de la próstata (HBP) y la prostatitis aguda, limitan su utilidad diagnóstica [2,3] (figura 1). Se ha encontrado que los hombres con niveles de PSA entre $4 \mathrm{ng} / \mathrm{mL}$ y $10 \mathrm{ng} / \mathrm{mL}$ pueden tener un $30 \%$ a $35 \%$ de probabilidad de una biopsia positiva para carcinoma de próstata, y de manera alterna, del 15\% al 30\% de todos los casos de cáncer de próstata pueden tener un nivel de PSA por debajo de $4 \mathrm{ng} / \mathrm{mL}$; además, su valor predictivo positivo relativamente bajo conlleva hasta un $70 \%$ de biopsias innecesarias [3-6]. Los niveles de PSA $>10 \mathrm{ng} / \mathrm{mL}$ solo muestran una probabilidad del $67 \%$ de un cáncer detectable por biopsia; sin embargo, la determinación de PSA es de gran utilidad para el monitoreo de la prostatectomía radical, ya que la remoción completa de la próstata debe llevar a concentraciones de PSA no detectables después de 6 a 8 semanas [4]. El marcador tumoral ideal para el diagnóstico de cáncer de próstata debe tener una alta sensibilidad y especificidad, que no solo

\footnotetext{
${ }^{1}$ Bacterióloga y Laboratorista Clínica. MSc en Virología. Directora Científica, Editora Médica Colombiana S.A. Medellín, Colombia. E-mail: atoro@edimeco.com.

${ }^{2}$ Médica, Especialista en Hematología. Asistente Científica, Editora Médica Colombiana S.A. Medellín, Colombia.

${ }^{3}$ Microbióloga y Bioanalista. MSc en Microbiología y Bioanálisis con énfasis en Hematología. Coordinadora de Control y Aseguramiento de la Calidad, Laboratorio Clínico Hematológico S.A. Medellín, Colombia.

${ }^{4}$ Médico, Especialista en Medicina de Laboratorio y Patología Anatómica, Subespecialista en Hematopatología. Fellow, College of American Pathologists (FCAP). Director, Laboratorio Clínico Hematológico S.A. Director, Editora Médica Colombiana S.A. Medellín, Colombia.
} 


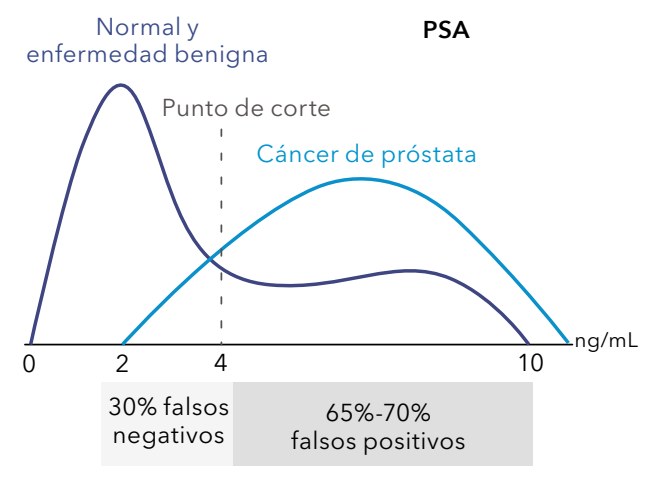

Figura 1. Comportamiento del PSA que demuestra su baja sensibilidad y especificidad.

logre diferenciar tejido prostático normal del tejido prostático maligno, sino que además pueda diferenciar los tumores de bajo grado de los más agresivos, a la vez que tenga la capacidad de identificar la mayoría de individuos con cáncer de próstata de manera temprana.

Teniendo en cuenta que el PSA es un marcador específico de órgano y no específico de cáncer, y con el fin de mejorar la sensibilidad y especificidad del PSA, se han venido desarrollando otras pruebas complementarias como la fracción libre de PSA (fPSA, del inglés, Free Specific Prostate Antigen) y el porcentaje de PSA libre (\%fPSA=[fPSA/PSA]x100), y más recientemente, el [-2]pro-PSA (p2PSA) y el Índice de Salud Prostática (phi, del inglés, Prostate Health Index) [7]. En el cáncer de próstata se presenta una disminución del \%fPSA, y se ha encontrado que la determinación del \%fPSA aumenta la sensibilidad y especificidad del PSA para su detección, reduciendo a su vez biopsias innecesarias. Por ejemplo, con un resultado de PSA entre $4 \mathrm{ng} / \mathrm{mL}$ y $10 \mathrm{ng} / \mathrm{mL}$, y un \%fPSA $\leq 10 \%$, se aumenta la probabilidad de detección de cáncer por biopsia a un 56\%, dependiendo del grupo de edad, en tanto que con un resultado de $\%$ fPSA $>25 \%$, la probabilidad disminuye a 8\% [8] (tabla 1). En general, se considera que el cáncer de próstata se asocia con un PSA aumentado y un fPSA o \%fPSA bajo.

El fPSA en suero se encuentra de varias formas; como ProPSA (precursor del PSA), BPSA (PSA "benigno", asociado a HBP) y como iPSA (PSA intacto) [10]. Con la intención de mejorar aún más la capacidad de las pruebas relacionadas con el PSA para diferenciar el cáncer de próstata de las enfermedades prostáticas benignas, y como resultado reducir el número de biopsias innecesarias, recientemente se estudió una de las formas truncadas del ProPSA, el [-2]proPSA [11], el cual es la isoforma con mayor especificidad en la detección de cáncer de próstata, ya que se origina principalmente en el epitelio prostático maligno y no en tejidos hiperplásicos transicionales, y ha demostrado su capacidad de diferenciar los tumores clínicos de bajo grado de los más agresivos que necesitan tratamiento [5]. Se ha encontrado que el [-2]proPSA representa hasta el 95\% del fPSA en los hombres con cáncer de próstata, en comparación con solo el $19 \%$ en los hombres con biopsias negativas [12].

Clínicamente, el [-2]proPSA, junto con el PSA total y el fPSA, se utiliza para calcular el Índice de Salud Prostática (phi)

\begin{tabular}{cc}
\hline $\begin{array}{c}\text { Tabla 1. Porcentaje de PSA libre (\%fPSA) y } \\
\text { probabilidad de cáncer de próstata en hom- } \\
\text { bres con valores de PSA total entre } 4 \mathrm{ng} / \mathrm{mL} \text { y } \\
10 \mathrm{ng} / \mathrm{mL}[8,9]\end{array}$ \\
\hline $\begin{array}{c}\text { PSA libre } \\
\text { (\%fPSA) }\end{array}$ & $\begin{array}{c}\text { Probabilidad de } \\
\text { cáncer }\end{array}$ \\
\hline$\leq 10 \%$ & $56 \%$ \\
$11 \%-15 \%$ & $28 \%$ \\
\hline $16 \%-20 \%$ & $20 \%$ \\
\hline $21 \%-25 \%$ & $16 \%$ \\
\hline$>25 \%$ & $8 \%$ \\
\hline
\end{tabular}


desarrollado por Beckman Coulter, y aprobado por la FDA en 2012 [13]. El phi se obtiene de la fórmula matemática ([-2]proPSA/fPSA)x/PSA, y ha demostrado una mayor utilidad clínica en cuanto a exactitud diagnóstica y discriminación entre cáncer agresivo (puntaje de Gleason $\geq 7$ ) y no agresivo, que el PSA y sus derivados [14,15] (tabla 2). Se ha encontrado que utilizando un punto de corte de phi $\geq 24$ en pacientes programados para biopsia, se puede evitar el $40 \%$ de dichas biopsias, y se dejarían de detectar solo un $5 \%$ de casos de cáncer de próstata clínicamente relevantes $[16,17]$, en tanto que un phi $>55$ se asocia con más del $50 \%$ de probabilidad de detectar un cáncer de próstata en la biopsia [18]. En general, se estima que la determinación de phi ayuda a clasificar los pacientes que requieren biopsia cuando tienen un PSA entre $4 \mathrm{ng} / \mathrm{mL}$ y $10 \mathrm{ng} / \mathrm{mL}$ y un examen rectal sin hallazgos sospechosos, teniendo también en cuenta que a mayor phi, mayor probabilidad de encontrar un cáncer más agresivo (mayor puntaje en la escala de Gleason) $[6,19,20]$.

En resumen, el phi es una prueba que considera varios parámetros de forma simultánea para evaluar el riesgo de cáncer de próstata, está aprobada por la FDA y recomendada en las guías de la NCCN (National Comprehensive Cancer Network) para la detección temprana de cáncer. Ha demostrado, además, ser una herramienta importante para reducir las biopsias innecesarias, con una exactitud diagnóstica superior a la del PSA y \%fPSA. En la figura 2 se observa un algoritmo para el uso del PSA y sus derivados en el diagnóstico de cáncer de próstata.

\section{Nota importante}

Es de anotar que las tres pruebas utilizadas para el cálculo del phi, el PSA,

Tabla 2. Odds ratio (OR), área bajo la curva (AUC) y valor $p$ de los diferentes marcadores para diferenciar la presencia o no de cáncer de próstata en hombres con valores de PSA total entre 4 $\mathrm{ng} / \mathrm{mL}$ y $10 \mathrm{ng} / \mathrm{mL}[21]$

\begin{tabular}{cccc}
\hline Marcador & OR & AUC & Valor $\boldsymbol{p}$ \\
\hline$[-2]$ proPSA & $0,99(0,96-1,02)$ & $0,48(0,38-0,57)$ & 0,694 \\
PSA & $0,87(0,71-1,06)$ & $0,59(0,50-0,68)$ & 0,181 \\
\%fPSA & $1,12(1,06-1,19)$ & $0,72(0,63-0,80)$ & $<0,001$ \\
phi & $0,93(0,91-0,96)$ & $0,76(0,69-0,84)$ & $<0,001$ \\
\hline
\end{tabular}

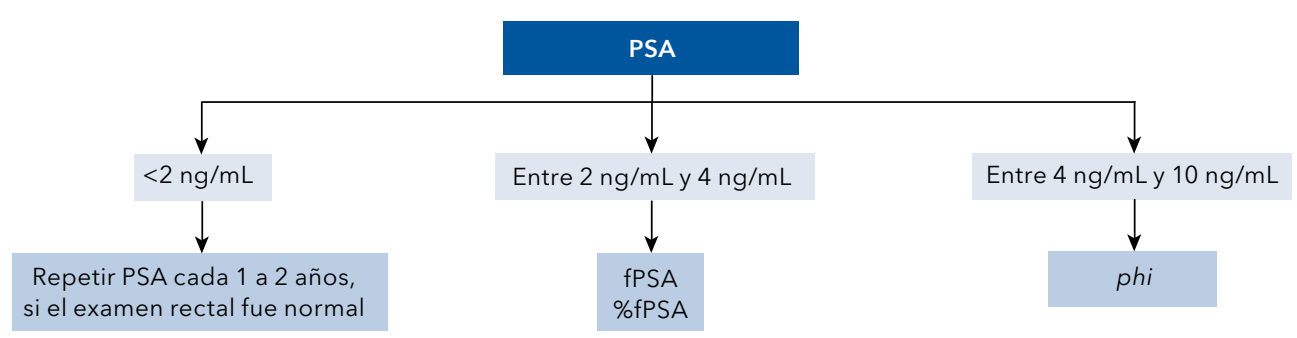

Figura 2. Algoritmo sugerido para las pruebas de tamizaje y detección de cáncer de próstata. 
el [-2]proPSA y el fPSA, se deben procesar en la misma muestra de suero y en el mismo analizador, para que el phi sea calculado automáticamente por el analizador. Hacer el cálculo manual con resultados de diferentes técnicas traería como consecuencia valores falsos, ya que cada técnica maneja sus propios puntos de corte que establece con su panel de sueros control y que corre con cada muestra, además, el phi ha sido validado para uso clínico teniendo en cuenta el desempeño individual y combinado de cada uno de los analitos medidos para el cálculo. Se recomienda entonces que cada laboratorio reporte sus resultados especificando la tecnología utilizada para las tres pruebas, y los valores de referencia de acuerdo con la misma. De igual forma, se debe tener en cuenta que las concentraciones de PSA y fPSA en los resultados van a depender del tipo de calibración que se utilizó; si se hizo de acuerdo con los estándares de calibración establecidos por la OMS o con los propios del analizador, ya que se ha encontrado que los resultados que se obtienen de pruebas calibradas de acuerdo con la OMS, arrojan resultados hasta $25 \%$ más bajos [22-24]. Por ejemplo, para conseguirse un mismo perfil de sensibilidad y especificidad, un punto de corte para el PSA de 3,1 $\mathrm{ng} / \mathrm{mL}$, de acuerdo con la calibración de la OMS, corresponde a uno de 4 $\mathrm{ng} / \mathrm{mL}$ con las técnicas calibradas tradicionalmente $[25,26]$ (tabla 3).

Desde 2018, la OMS diseñó un nuevo estándar internacional para PSA total y fPSA (estándar 17/100). La aplicación de pruebas de laboratorio ofrecidas por fabricantes que tengan trazabilidad con este estándar internacional, es altamente importante para poder comparar resultados entre laboratorios. De lo contrario, la comparación de resultados entre los diferentes laboratorios constituye un riesgo en el seguimiento de los pacientes, y puede limitar la aplicación de los resultados [27].

\begin{tabular}{|c|c|c|}
\hline $\begin{array}{c}\text { PSA } \\
\text { (Hybritech) }\end{array}$ & PSA (OMS) & $\begin{array}{l}\text { Probabilidad } \\
\text { de cáncer }\end{array}$ \\
\hline $0-2 \mathrm{ng} / \mathrm{mL}$ & $0-1,6 \mathrm{ng} / \mathrm{mL}$ & $1 \%$ \\
\hline $2-4 \mathrm{ng} / \mathrm{mL}$ & $1,6-3,1 \mathrm{ng} / \mathrm{mL}$ & $15 \%$ \\
\hline $4-10 \mathrm{ng} / \mathrm{mL}$ & $3,1-7,8 \mathrm{ng} / \mathrm{mL}$ & $25 \%$ \\
\hline$>10 \mathrm{ng} / \mathrm{mL}$ & $>7,8 \mathrm{ng} / \mathrm{mL}$ & $>50 \%$ \\
\hline
\end{tabular}

\section{[-2]proPSA (p2PSA, Access Hybritech p2PSA) [-2]proPSA (p2PSA, Access Hybritech p2PSA)}

\section{Utilidad clínica de la prueba}

El [-2]proPSA es la isoforma del fPSA con mayor especificidad en la detección de cáncer de próstata. Es una prueba que utiliza la tecnología de Access Hybritech para la determinación cuantitativa de [-2]proPSA en sangre. La finalidad de su determinación es usar este valor, en combinación con el PSA y el fPSA, para 
calcular el Índice de Salud Prostática (phi), el cual ayuda a diferenciar entre enfermedades prostáticas benignas y cáncer de próstata, en particular en los pacientes con un PSA total entre $4 \mathrm{ng} / \mathrm{mL}$ y $10 \mathrm{ng} / \mathrm{mL}$, y sin hallazgos sospechosos en el examen rectal.

\section{Fundamento de la prueba}

El Access Hybritech p2PSA ([-2]pro-PSA) es un ensayo inmunoenzimático de tipo "sándwich" (figura 3). La muestra de suero del paciente se agrega a un recipiente de reacción que contiene un anticuerpo monoclonal de ratón anti-PSA marcado con fosfatasa alcalina, y partículas paramagnéticas recubiertas con otro anticuerpo monoclonal de ratón anti-[-2]proPSA que reacciona con diferentes sitios antigénicos del [-2]proPSA. El [-2]proPSA que está en la muestra del paciente, se une a ambos anticuerpos durante un periodo de incubación. Luego, se hacen lavados que garanticen que solo permanezcan en el recipiente de reacción los complejos

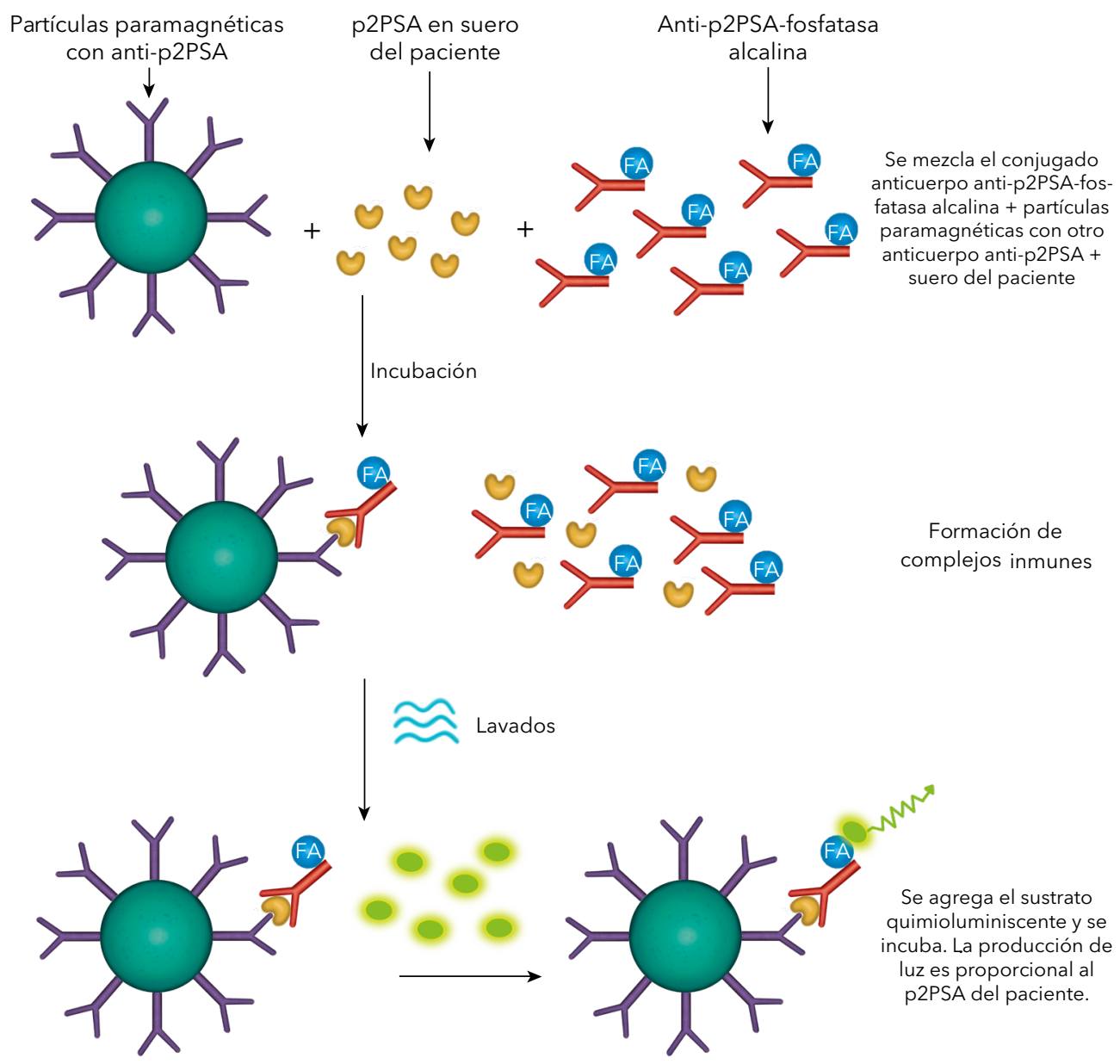

Figura 3. Técnica inmunoenzimática tipo sándwich para la detección de p2PSA en suero de pacientes. 
inmunes unidos a la fase sólida que se mantienen en un campo magnético, mientras que los componentes no unidos se eliminan. Posteriormente, se agrega un sustrato quimioluminiscente (Lumi-Phos 530) al recipiente de incubación, y la luz generada por la reacción se mide con un luminómetro. La producción de luz es directamente proporcional a la concentración de [-2]proPSA en la muestra del paciente.

\section{Preparación del paciente}

El paciente debe estar preferiblemente en ayunas. La prueba no debe realizarse si hubo manipulación prostática previa, como examen rectal digital, masaje prostático, ultrasonido transrectal, prostatitis o biopsia prostática, debido a que puede haber un aumento transitorio en los niveles de [-2]proPSA, fPSA y PSA. En la tabla 4 se indican los tiempos de espera según el procedimiento realizado.

\section{Muestra y almacenamiento}

El suero es la muestra adecuada para los análisis Access Hybritech [-2]proPSA, PSA y fPSA. No se deben utilizar muestras de plasma y se debe evitar la hemólisis. Es necesario que la muestra se coagule completamente antes de separar el suero por centrifugación. En caso de que la muestra no se procese en las primeras 3 horas, se puede conservar en refrigeración $\left(2^{\circ} \mathrm{C}\right.$ a $8{ }^{\circ} \mathrm{C}$ ) por 24 horas. Las muestras que serán almacenadas hasta por 5 meses deben congelarse a $-20^{\circ} \mathrm{C}$, y si se van a almacenar por más tiempo, deben congelarse a $-70^{\circ} \mathrm{C}$. Los ciclos repetidos de congelación y descongelación no tienen efecto en el [-2]proPSA, PSA ni fPSA; sin embargo, se recomien- da volver a congelar rápidamente las muestras descongeladas.

\section{Limitaciones de la prueba}

El uso de fármacos inhibidores de la enzima 5- $\alpha$-reductasa generalmente disminuye los niveles del PSA, fPSA y [-2]proPSA en los pacientes. También se deben evaluar cuidadosamente los resultados de pacientes sospechosos de tener niveles altos de proteína total (>8 g/dL). Los resultados del phi deben interpretarse en el contexto clínico del paciente, incluyendo síntomas, historia clínica, datos de pruebas adicionales y cualquier otra información relacionada. Los resultados no deben interpretarse como evidencia absoluta de la presencia o ausencia de cáncer de próstata; la biopsia es la única prueba que puede confirmar el diagnóstico de cáncer de próstata.

No es posible cuantificar con precisión concentraciones de PSA total $<0,008$ $\mathrm{ng} / \mathrm{mL}$ y de PSA libre $<0,005 \mathrm{ng} / \mathrm{mL}$.

Tabla 4. Procedimientos y tiempos de espera sugeridos, previos a la toma de la muestra [28-30]

\begin{tabular}{lc}
\hline Procedimiento & $\begin{array}{c}\text { Tiempo de espera } \\
\text { recomendado }\end{array}$ \\
\hline Masaje prostático & 1 semana \\
\hline Tacto rectal & días a 1 semana \\
Cistoscopia & 7 días \\
$\begin{array}{l}\text { Ultrasonido } \\
\text { transrectal } \\
\text { Resección } \\
\text { transuretral de la } \\
\text { próstata } \\
\text { Biopsia prostática }\end{array} \quad 6$ semanas \\
Ecografía transrectal & 6 semanas \\
Actividad sexual & 3 días \\
Bicicleta y equitación & 24 horas \\
\hline
\end{tabular}




\section{Referencias}

1. World Health Organization (WHO). Incidence, mortality and prevalence by cancer site. The Global Cancer Observatory (GLOBOCAN); 2020. p. 1-2. Acceso 06 de abril de 2021. Disponible en https://gco.iarc.fr/today/ data/factsheets/populations/170-colombiafact-sheets.pdf.

2. Carroll PR, Parsons JK, Andriole G, Bahnson RR, Castle EP, Catalona WJ, et al. NCCN Guidelines Insights: Prostate Cancer Early Detection, Version 2.2016. J Natl Compr Canc Netw 2016;14:509-519. https://doi.org/10.6004/ jnccn.2016.0060.

3. Thompson IM, Ankerst DP, Chi C, Lucia MS, Goodman PJ, Crowley JJ, et al. Operating characteristics of prostate-specific antigen in men with an initial PSA level of $3.0 \mathrm{ng} / \mathrm{ml}$ or lower. JAMA 2005;294:66-70. https://doi. org/10.1001/jama.294.1.66.

4. National Comprehensive Cancer Network (NCCN). NCCN Clinical Practice Guidelines in Oncology: Prostate cancer early detection. Plymouth Meeting, USA: 2018. p. 1-52. Acceso 02 de abril de 2021. Disponible en https:// www2.tri-kobe.org/nccn/guideline/urological/ english/prostate_detection.pdf.

5. Vukovic I, Djordjevic D, Bojanic N, Babic U, Soldatovic I. Predictive value of [-2]proPSA (P2PSA) and its derivatives for the prostate cancer detection in the 2.0 to $10.0 \mathrm{ng} / \mathrm{mL}$ PSA range. International Braz J Urol 2017;43:4856. https://doi.org/10.1590/S1677-5538. IBJU.2016.0256.

6. White J, Shenoy BV, Tutrone RF, Karsh LI, Saltzstein DR, Harmon WJ, et al. Clinical utility of the Prostate Health Index (phi) for biopsy decision management in a large group urology practice setting. Prostate Cancer Prostatic Dis 2018;21:78-84. https://doi.org/10.1038/ s41391-017-0008-7.

7. Boegemann M, Stephan C, Cammann H, Vincendeau S, Houlgatte A, Jung $K$, et al. The percentage of prostate-specific antigen (PSA) isoform [-2]proPSA and the Prostate Health Index improve the diagnostic accuracy for clinically relevant prostate cancer at initial and repeat biopsy compared with total PSA and percentage free PSA in men aged $\leq 65$ years. BJU International 2016;117:72-79. https://doi. org/10.1111/bju.13139.

8. Jain S, Pincus MR, Bluth MH, McPherson RA, Bowne WB, Lee P. Diagnosis and management of cancer using serologic and other body fluid markers. In: Pincus MR, Bluth MH, McPherson RA, Bowne WB, eds. Henry's Clinical Diagnosis and Management by Laboratory Methods. 23rd ed. St. Louis: Elsevier; 2017. p. 1442-1444.

9. Algeciras-Schimnich A. Insights: The Prostate Health Index (phi) in prostate cancer risk assessment. Minnesota, USA: Mayo Clinic Laboratories; 2017. Department of Laboratory Medicine and Pathology. Acceso 11 de mayo de 2021. Disponible en https://news.mayocliniclabs.com/2017/08/14/prostate-health-indexphi-prostate-cancer-risk-assessment-hot-topic/.

10. Özen H, Sözen S. PSA isoforms in prostate cancer detection. Eur Urol Supp 2006;5:495-499. https://doi.org/10.1016/j.eursup.2006.02.017.

11. Filella $X$, Foj L, Alcover J, Augé JM, Escudero JM, Molina R. ProPSA, un nuevo biomarcador para la detección y el manejo del cáncer de próstata. Rev Laboratorio Clín 2013;6:75-81. https://doi.org/10.1016/j.labcli.2013.01.006.

12. Mikolajczyk SD, Marker KM, Millar LS, Kumar A, Saedi MS, Payne JK, et al. A truncated precursor form of prostate-specific antigen is a more specific serum marker of prostate cancer. Cancer Res 2001;61:6958-6963.

13. U.S. Food \& Drug Administration. Quantitative test for determination of [-2]proPSA levels. Silver Spring: FDA Summary of Safety and Effectiveness Data; 2012. p. 1-32. Acceso 15 de mayo de 2021. Disponible en https://www.accessdata.fda.gov/cdrh_docs/pdf9/P090026b.pdf.

14. Tosoian JJ, Druskin SC, Andreas D, Mullane P, Chappidi M, Joo S, et al. Use of the Prostate Health Index for detection of prostate cancer: results from a large academic practice. Prostate Cancer Prostatic Dis 2017;20:228-233. https:// doi.org/10.1038/pcan.2016.72.

15. Loeb S, Bruinsma SM, Nicholson J, Briganti A, Pickles T, Kakehi Y, et al. Active surveillance for prostate cancer: a systematic review of clinicopathologic variables and biomarkers for risk stratification. Eur Urol 2015;67:619-626. https://doi.org/10.1016/j.eururo.2014.10.010. 
16. de la Calle C, Patil D, Wei JT, Scherr DS, Sokoll L, Chan DW, et al. Multicenter evaluation of the Prostate Health Index to detect aggressive prostate cancer in biopsy naive men. J Urol 2015;194:65-72. https://doi. org/10.1016/j.juro.2015.01.091.

17. Osses DF, Remmers S, Schroder FH, van der Kwast T, Roobol MJ. Results of prostate cancer screening in a unique cohort at $19 \mathrm{yr}$ of followup. Eur Urol 2019;75:374-377. https://doi. org/10.1016/j.eururo.2018.10.053.

18. Abrate A, Lughezzani G, Gadda GM, Lista G, Kinzikeeva E, Fossati N, et al. Clinical use of [-2]proPSA (p2PSA) and its derivatives (\%p2PSA and Prostate Health Index) for the detection of prostate cancer: A review of the literature. Korean J Urol 2014;55:436-445. https://doi. org/10.4111/kju.2014.55.7.436.

19. Heidegger I, Klocker H, Pichler R, Pircher A, Prokop W, Steiner E, et al. ProPSA and the Prostate Health Index as predictive markers for aggressiveness in low-risk prostate cancerresults from an international multicenter study. Prostate Cancer Prostatic Dis 2017;20:271-275. https://doi.org/10.1038/pcan.2017.3.

20. Catalona WJ, Partin AW, Sanda MG, Wei JT, Klee GG, Bangma CH, et al. A multicenter study of [-2]pro-prostate specific antigen combined with prostate specific antigen and free prostate specific antigen for prostate cancer detection in the 2.0 to $10.0 \mathrm{ng} / \mathrm{ml}$ prostate specific antigen range. J Urol 2011;185:1650-1655. https://doi.org/10.1016/j.juro.2010.12.032.

21. Shore ND, Pieczonka CM, Henderson RJ, Bailen JL, Saltzstein DR, Concepcion RS, et al. A comparison of prostate health index, total PSA, \%free PSA, and proPSA in a contemporary US population-The MiCheck-01 prospective trial. Urol Oncol 2020;38:683.e681-683.e610. https://doi.org/10.1016/j.urolonc.2020.03.011.

22. Boegemann $M$, Arsov $C$, Hadaschik B, Herkommer K, Imkamp F, Nofer JR, et al. Discordant prostate specific antigen test results despite WHO assay standardization. Int J Biol Markers 2018;33:275-282. https://doi. org/10.1177/1724600818754750.

23. Stephan $C$, Bangma $C$, Vignati G, Bartsch G, Lein $\mathbf{M}$, Jung $\mathbf{K}$, et al. 20-25\% lower concentrations of total and free prostate-specific antigen (PSA) after calibration of PSA assays to the WHO reference materials--analysis of 1098 patients in four centers. Int J Biol Markers 2009;24:65-69.

24. Vignati G, Giovanelli L. Standardization of PSA measures: a reappraisal and an experience with WHO calibration of Beckman Coulter Access Hybritech total and free PSA. Int J Biol Markers 2007;22:295-301.

25. Stephan $C$, Kopke $T$, Semjonow A, Lein $M$, Deger S, Schrader M, et al. Discordant total and free prostate-specific antigen (PSA) assays: does calibration with WHO reference materials diminish the problem? Clin Chem Lab Med 2009;47:1325-1331. https://doi.org/10.1515/ CCLM.2009.285.

26. Foj L, Filella $X$, Alcover J, Auge JM, Escudero JM, Molina R. Variability of assay methods for total and free PSA after WHO standardization. Tumour Biol 2014;35:1867-1873. https://doi. org/10.1007/s13277-013-1249-2.

27. Ferguson J, Atkinson E, Rigsby $\mathbf{P}$, Burns $\mathbf{C}$. WHO International Collaborative Study of the proposed 2nd WHO is for total PSA (PSA-ACT + free PSA). Geneva, Switzerland: World Health Organization (WHO). Expert committee on biological standardization; 2018. Acceso 03 de abril de 2021. Disponible en https://www.who. int/biologicals/BS.2018.2340_PSA_Total.pdf.

28. Prostate Cancer UK. The test PSA. London, UK: Prostate Cancer UK; 2020. Acceso 19 de mayo de 2021. Disponible en https://prostatecanceruk. org/prostate-information/prostate-tests/psa-test.

29. Pagana KD, Pagana TJ. Mosby's Manual of Diagnostic and Laboratory Test. 4th ed. Misuri, USA; Mosby Elsevier; 2010.

30. Bossens MM, Van Straalen JP, De Reijke TM, Kurth KH, Sanders GT. Kinetics of prostatespecific antigen after manipulation of the prostate. Eur J Cancer 1995;31a:682-685. https:// doi.org/10.1016/0959-8049(95)00016-c. 